\title{
Nonlinear response to ultrasound of encapsulated microbubbles
}

\author{
J. Jiménez-Fernández
}

\begin{abstract}
A B S T R A C T
The acoustic backscatter of encapsulated gas-filled microbubbles immersed in a weak compressible liquid and irradiated by ultrasound fields of moderate to high pressure amplitudes is investigated theoretically. The problem is formulated by considering, for the viscoelastic shell of finite thickness, an isotropic hyperelastic neo-Hookean model for the elastic contribution in addition to a Newtonian viscous component. First and second harmonic scattering cross-sections have been evaluated and the quantitative influence of the driving pressure amplitude on the harmonic resonance frequencies for different initial equilibrium bubble sizes and for different encapsulating physical properties has been determined. Conditions for optimal second harmonic imaging have been also investigated and some regions in the parameters space where the second harmonic intensity is dominant over the fundamental have been identified. Results have been obtained for albumin, lipid and polymer encapsulating shells, respectively.
\end{abstract}

\section{Introduction}

Gas-filled microbubbles stabilized against dissolution by lipid, polymeric or albumin shells, irradiated by an ultrasound field, enhance the acoustic backscattering from blood-filled regions and, hence, improve diagnostic ultrasound imaging [1,2]. More recently they also have been used for targeted therapeutic gene and drug delivery [3]. In the above applications, a precise knowledge of the acoustic properties of the backscatter signal is essential, mainly for ultrasound fields of moderate to high pressure amplitudes for which microbubbles are driven into nonlinear radial oscillations. As a consequence of this nonlinear behavior, the scattered signal is composed by integer multiples (harmonics) of the transmitted frequency. This harmonic content is the basis of diagnostic techniques known under the generic term of harmonic imaging which rely on transmitting an incident signal at a fundamental frequency and filtering the returned echoes at higher harmonic frequencies, specially the corresponding to the second harmonic that besides the fundamental is the harmonic of highest intensity. Therefore, a quantitative analysis of the harmonic intensities as well as the harmonic resonance frequencies is required.

To fulfil the above purposes, the dynamic and sound emission of a single bubble must be investigated. Usually, the dynamics of encapsulated gas bubbles irradiated by sound fields has been studied following a generalized Rayleigh-Plesset approach, by including in the analysis elastic and viscous properties of the encapsulating layer. In pioneering works $[4,5]$, these mechanical properties of the layer were introduced by adding to the usual surface tension coefficient, shell elasticity and shell friction terms in the normal stress condition at the gas liquid interface. A general analysis considering a viscoelastic layer of finite thickness was performed by Church [6]. In this basic work, where the shell was modeled by means of the Kelvin-Voigt rheological equation, it was established that the resonance frequency of individual microbubbles increases approximately as the square of the modulus of elasticity of the shell. The Church model was subsequently applied by Hoff et al. [7] to bubbles encapsulated by polymeric shells. It was found that the resonance frequency is about four times higher than the one corresponding to a free bubble of the same radius. The dynamics of encapsulated gas bubbles surrounded by a compressible viscoelastic fluid was investigated by Khismatullin and Nadim [8]. Their results show the strong influence of viscous damping on resonance frequency which produces significant divergences from the undamped natural frequency. Additional results concerning the influence of viscous damping and harmonic resonance frequencies were obtained later by Khismatullin [9].

The assumption of a homogeneous and isotropic layer has been discussed by Chatterjee and Sarkar [10], and Sarkar et al. [11]. They have proposed a different approach based on interfacial models with intrinsic surface rheology. Similar predictions about resonances are found, i.e., the encapsulation increases the resonant frequency. A nonlinear extension of these interfacial elasticity models has been recently developed [12]. In the model of Stride [13], it is assumed that the bubble is encapsulated by a homogeneous molecular monolayer with surface tension and interfacial viscosity depending on the surface molecular concentration. Numerical results obtained from this model show a clear influence of coating properties on the resonance frequency. 
For small acoustic amplitudes some theoretical predictions concerning the quantitative influence of the viscoelastic properties of the shell on the resonance frequency have been experimentally confirmed. In particular, optical experiments carried out by Van der Meer et al. [14] show that, in comparison with a free uncoated bubble, shell elasticity increases the resonance frequency by about $50 \%$, shell viscosity being the first damping factor. Recently, experiments on phospholipid-coated contrast agents performed by Overvelde et al. [15] have shown a significant decrease of the frequency corresponding to the maximum response with increasing pressure and a pronounced skewness of the resonance curve. It is surprising, however, that these nonlinear responses are observed for acoustic pressures as low as $10 \mathrm{kPa}$. These experimental results have been appropriately described by the model proposed by Marmottant et al. [16], a heuristic model based on the behavior of phospholipid monolayer coating, with surface tension depending on bubble area, which takes into account shell buckling and rupture. This model predicts other characteristic nonlinear responses of lipid coated bubbles observed for small acoustic pressures like the one termed "compression-only" behavior [17].

The problem for high acoustic amplitudes has been less explored. A numerical study of the dependence of the resonance frequency, defined as the driving frequency which maximizes the scattering cross-section of the backscatter signal, on the pressure amplitude in the range 0.1-1 MPa, was performed by MacDonald et al. [18]. Their calculations show a shift of the resonant frequency values to lower frequencies as the pressure amplitude increases. For large bubbles the discrepancies between linear and nonlinear values may reach even $40 \%$. In this analysis the problem was formulated by means of the general Keller-Herring equation modified to incorporate viscoelastic properties of the shell. A model previously developed by Morgan et al. [19], was also used by Wu et al. [20] in a theoretical study on the nonlinear properties of encapsulated bubbles. In this work it was also confirmed that the frequency at which a peak of the radial oscillation occurs is a decreasing function of the driving acoustic amplitude pulse. In the regime of nonlinear oscillations, results obtained by Doinikov et al. [21] for lipid-shelled microbubbles, confirm a decrease in resonance frequencies with increasing acoustic pressure.

A basic aspect in the theoretical analysis of bubble dynamics in the nonlinear regime is the formulation of the governing equations with an appropriate constitutive equation for the encapsulating shell. In this sense, it must be remarked that the Kelvin-Voigt model is restricted to infinitesimal displacements and velocity gradients, consequently, such a model should be limited to small amplitudes of the external pressure fields. For bubbles coated by shells of finite thickness, some approaches including nonlinear constitutive equations to describe the rheological behavior of the shell have also been considered. The constitutive equation of a neo-Hookean material has been discussed by Allen and Rashid [22] and Jiménez-Fernández [23]. The more general Mooney-Rivlin constitutive law along with the Skalak and Kelvin-Voigt models were investigated by Tsiglifis and Pelekasis [24] in order to describe strain-softening as well as strain-hardening behaviors of membranes following the approach used in the study of biological cells. It was concluded that if the membrane is strain-softening, as it is predicted by the Mooney-Rivlin equation, the resonance frequency decreases with increasing sound amplitude. The Mooney-Rivlin model has been also used to predict the behavior observed in acoustic experiments on lipid-shelled Definity microbubbles [25]. For lipid coating, the equation of a viscoelastic Maxwell fluid has been considered by Doinikov and Dayton [26].

In this work, the scattering of gas bubbles encapsulated by a viscoelastic shell of finite thickness immersed in a liquid and subject to driving acoustic fields of high amplitudes, is studied
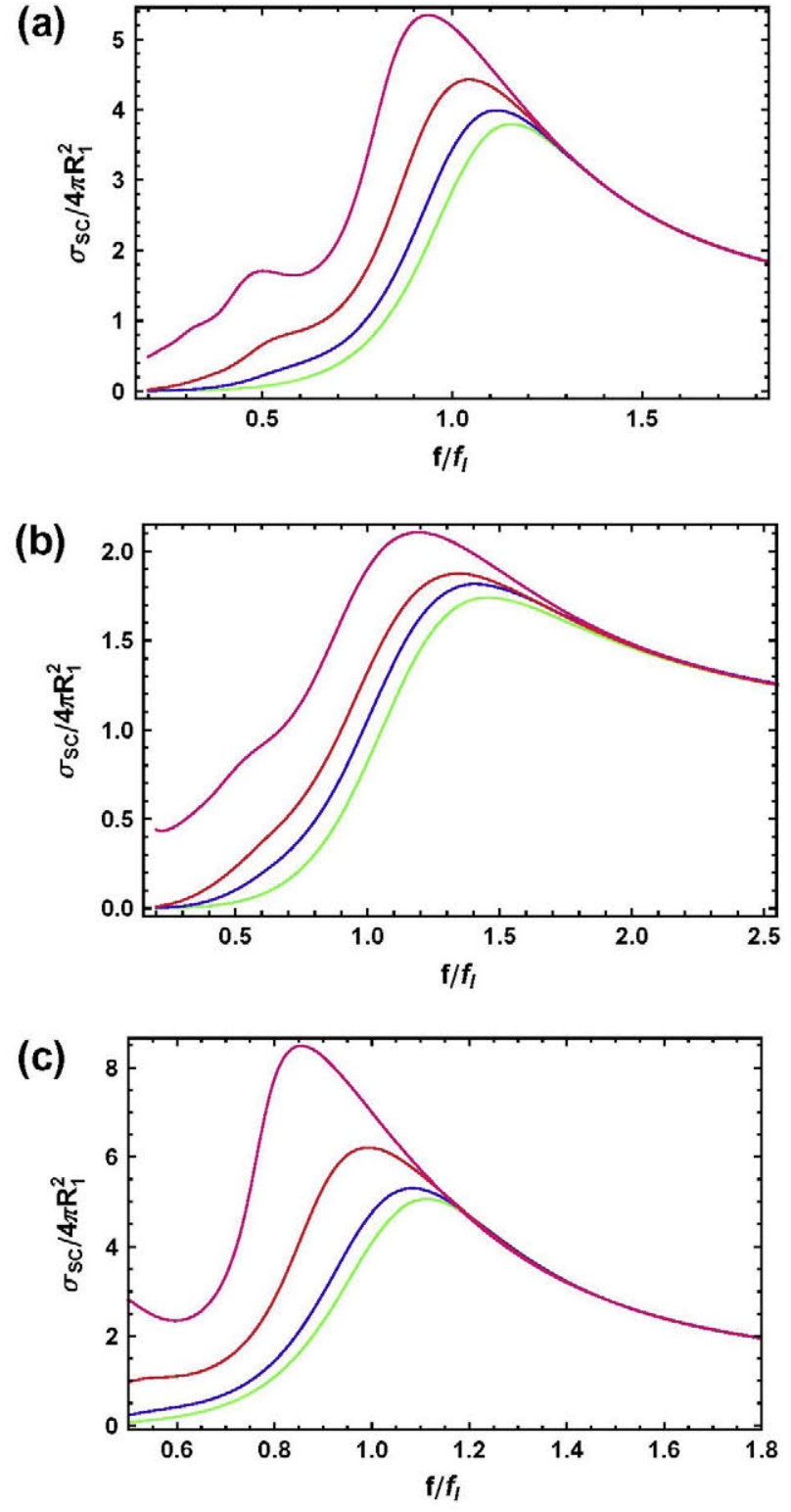

Fig. 1. Scattering cross-section normalyzed with $4 \pi R_{1}^{2}$ versus driving frequency normalyzed with the linear resonance frequency $f_{l}$ for: (a) albumin-shelled bubbles in water: initial bubble radius $R_{1}=3 \mu \mathrm{m}$, pressure amplitudes: $p_{A}=300 \mathrm{kPa}$ (blue), $p_{A}=500 \mathrm{kPa}$ (red), $p_{A}=700 \mathrm{kPa}$ (pink). (b) Polymer-shelled bubbles in blood: initial bubble radius $R_{1}=4 \mu \mathrm{m}$, pressure amplitudes: $p_{A}=500 \mathrm{kPa}$ (blue), $p_{A}=700 \mathrm{kPa}$ (red), $P_{A}=1000 \mathrm{kPa}$ (pink), (c) lipid-shelled bubbles in water: initial bubble radius $R_{1}=1.5 \mu \mathrm{m}$, pressure amplitudes: $p_{A}=100 \mathrm{kPa}$ (blue), $p_{A}=200 \mathrm{kPa}$ (red), $P_{A}=300 \mathrm{kPa}$ (pink). In each graphic the green line corresponds to the linear first harmonic scattering-cross section $\sigma_{s 1}$. (For interpretation of the references to colour in this figure legend, the reader is referred to the web version of this article.)

theoretically. Emphasis has been focused to analyze the different harmonic components of the backscatter signal.

The paper is organized as follows. In Section 2, the equations for radial bubble oscillations are derived. The problem is formulated by considering for the viscoelastic shell, an isotropic hyperelastic neo-Hookean model for the elastic contribution, in addition to a viscous component. Because ultrasound fields of high pressure amplitude will be considered, high values of the bubble wall velocity are expected, consequently, compressibility effects in the liquid phase have been also included in the formulation. In Section 3, a linear analysis of the governing equations is carried out, based on which, analytical expressions for the resonance frequency as well 
(a)

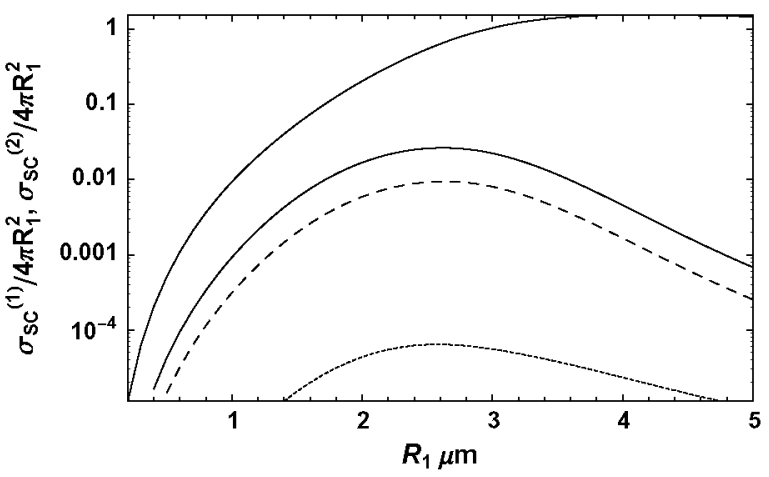

(b)

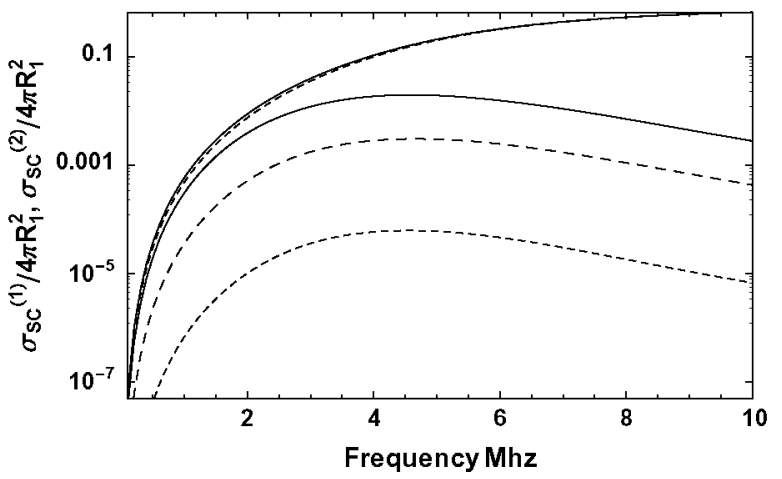

Fig. 2. (a) First and second-harmonic scattering cross-sections, $\sigma_{s c}^{(1)}$ and $\sigma_{s c}^{(2)}$ normalized with $4 \pi R_{1}^{2}$ for polymeric bubbles as functions of the initial inner bubble radius at a transmitted frequency $f=5 \mathrm{MHz}$. Blue line correspond to $\sigma_{s 1}$ and red lines correspond to $\sigma_{s c}^{(2)}: p_{A}=30 \mathrm{kPa}$ (dotted), $p_{A}=300 \mathrm{kPa}$ (dashed), $p_{A}=500 \mathrm{kPa}$ (dark). (b) First and second-harmonic scattering cross-sections, $\sigma_{s c}^{(1)}$ and $\sigma_{s c}^{(2)}$ normalized with $4 \pi R_{1}^{2}$ for polymeric bubbles as functions of the transmitted frequency for an initial inner bubble radius $R_{1}=2 \mu \mathrm{m}$ : blue lines correspond to $\sigma_{S 1}$ (dotted), and $\sigma_{S c}^{(1)}$ for $p_{A}=500 \mathrm{kPa}$ (dark). Red lines correspond to $\sigma_{s c}^{(2)}: p_{A}=30 \mathrm{kPa}$ (dotted), $p_{A}=200 \mathrm{kPa}$ (dashed), $p_{A}=500 \mathrm{kPa}$ (dark). (For interpretation of the references to colour in this figure legend, the reader is referred to the web version of this article.)

as for the (linear) first harmonic scattering cross-section have been determined. For finite pressure amplitudes, total, first and second scattering cross-sections have been evaluated as a function of the driven pressure amplitude for different initial bubble size and different encapsulating physical properties. From these, first and second harmonic resonance frequencies have been determined. Finally, some ranges of parameters where second harmonic imaging may be optimized, i.e., where the ratio of the second harmonic scattering cross-section to the fundamental is maximized have been identified. Numerical results have been obtained for albumin, lipid and polymer encapsulating shells.

\section{The mathematical model}

Let us consider a gas bubble encapsulated by a viscoelastic shell and immersed in a Newtonian liquid of density $\rho_{l}$ and viscosity $\eta_{l}$. In a spherical coordinate system $\{r, \theta, \phi\}$ with its origin at the center of the spherical bubble, the instantaneous inner and outer radii are denoted by $r_{1}$ and $r_{2}$ respectively. It is assumed that the volumetric bubble motion is purely radial so that spherical symmetry is held at any time. It is also considered that the encapsulating layer is incompressible and therefore:

$r_{2}^{3}-r_{1}^{3}=R_{2}^{3}-R_{1}^{3}$

where $R_{1}$ and $R_{2}$ are the initial inner and outer radii respectively. Integration of the motion equations in the solid shell, i.e. between $r_{1}$ and $r_{2}$, leads to the following equation:

$$
\rho_{s}\left[\varphi\left(r_{1}\right)-\varphi\left(r_{2}\right)\right]=T_{r r}^{s}\left(r_{2}\right)-T_{r r}^{s}\left(r_{1}\right)+\Gamma\left(r_{1}\right)
$$

where $\rho_{s}$ is the density of the shell, $T_{r r}^{s}$ is the $r r$-component of the stress tensor in the shell $\mathbf{T}^{\mathbf{s}}$, and $\varphi(r)$ is the potential:

$\varphi(r)=\frac{1}{r} \frac{\partial}{\partial t}\left(\dot{r}_{1} r_{1}^{2}\right)-\frac{\left(\dot{r}_{1} r_{1}^{2}\right)^{2}}{2 r^{4}}$

where point denotes differentiation with time and $\Gamma\left(r_{1}\right)$ is the integral:

$\Gamma=2 \int_{r_{1}}^{r_{2}} \frac{\tau_{r r}^{s}-\tau_{\theta \theta}^{s}}{r} d r$

$\tau_{r r}^{s}, \tau_{\theta \theta}^{s}$ are, respectively, the $r r$-component and the $\theta \theta$-component of the extra stress tensor $\tau^{s}=\mathbf{T}^{\mathbf{s}}+p^{s} \mathbf{I}$ in the shell, $p^{s}$ is the isotropic pressure and $\mathbf{I}$ is the identity tensor.

A normal stress balance at the internal interphase $r=r_{1}$ leads to:

$T_{r r}^{s}\left(r_{1}\right)=-p_{g}\left(r_{1}\right)+\frac{2 \sigma_{1}}{r_{1}}$

where $p_{g}=p_{g 0}\left(r_{1} / R_{1}\right)^{3 \gamma}$ is the gas pressure inside the bubble, $p_{g 0}$ the gas pressure at the initial state, $\gamma$ the polytropic index and $\sigma_{1}$ the surface tension coefficient at the gas-shell surface. Likewise, a stress balance at the external interphase $r=r_{2}$ gives:

$T_{r}^{s}\left(r_{2}\right)=T_{r r}^{l}\left(r_{2}\right)-\frac{2 \sigma_{2}}{r_{2}}$

where $\sigma_{2}$ is the surface tension coefficient at the shell-liquid surface and $T_{r r}^{l}$, the $r$-component of the stress tensor in the liquid $\mathbf{T}^{\mathbf{l}}$. $T_{r r}^{l}\left(r_{2}\right)$ must be determined by integration of the motion equations in the external liquid phase. In the present work, a boundary layer analysis analogous to those performed by Prosperetti and Lezzi [27] for a free bubble and by Khismatullin and Nadim [8] for an encapsulated bubble, has been carried out in order to include compressibility effects in the liquid phase. Accordingly, the following expression for $T_{r r}^{\mathrm{j}}\left(r_{2}\right)$ has been obtained:

$T_{r r}^{l}\left(r_{2}\right)=-4 \eta_{l} \frac{\dot{r}_{2}}{r_{2}}-\rho_{l} \varphi\left(r_{2}\right)-p_{\infty}+\frac{\rho_{l}}{c_{s}} \frac{d^{2}}{d t^{2}}\left(r_{2}^{2} \dot{r}_{2}\right)$

where $p_{\infty}=p_{0}+p_{A} \sin \omega t, p_{0}$ is the ambient pressure, $p_{A}$ the acoustic pressure amplitude, $\omega$ the angular frequency and $c_{s}$ the sound velocity in the liquid phase. By substitution of expressions (3)-(7) into expression (2) the following governing equation is obtained:

$$
\begin{aligned}
& \rho_{s} r_{1} \ddot{r}_{1}\left[1+\psi-\frac{\rho_{l}}{\rho_{s} c_{s}} \dot{r}_{1} \Phi\right] \\
& \quad+\rho_{s} \dot{r}_{1}^{2}\left[\frac{3}{2}+\psi\left(2-\frac{r_{1}^{3}}{2 r_{2}^{3}}\right)-\frac{\rho_{l}}{\rho_{s} c_{s}} \frac{1}{2} \frac{d}{d t} r_{1} \Phi\right] \\
& \quad=\left(p-p_{\infty}\right)+\frac{\rho_{l}}{\rho_{s} c_{s}} \frac{d}{d t} \frac{r_{1}}{1+\psi}\left(p-p_{\infty}\right)
\end{aligned}
$$

where

$\psi=\frac{\rho_{l}-\rho_{s}}{\rho_{s}} \frac{r_{1}}{r_{2}} ; \quad \Phi=\frac{1+\psi r_{1}^{3} / r_{2}^{3}}{1+\psi}$

and

$p=p_{g}-\left(\frac{2 \sigma_{1}}{r_{1}}+\frac{2 \sigma_{2}}{r_{2}}\right)-4 \eta_{l} \frac{\dot{r}_{2}}{r_{2}}+\Gamma\left(r_{1}\right)$

The function $\Gamma\left(r_{1}\right)$ defined by Eq. (4) is determined by the constitutive equation considered for the stress tensor $\tau^{s}$. In this work, it will be assumed that $\tau^{s}$ is composed by the addition of elastic and viscous contributions: $\tau_{e}^{s}$ and $\tau_{v}^{s}$. The elastic component $\tau_{e}^{s}$ satisfies the equation of an isotropic, incompressible, neo-Hookean hyperelastic solid: [28]

$\tau_{e}^{s}=2 G B$ 
(a)

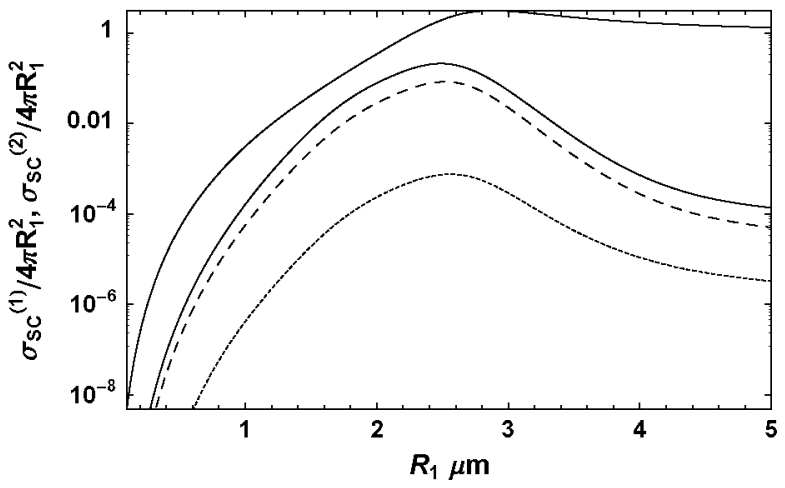

(b)

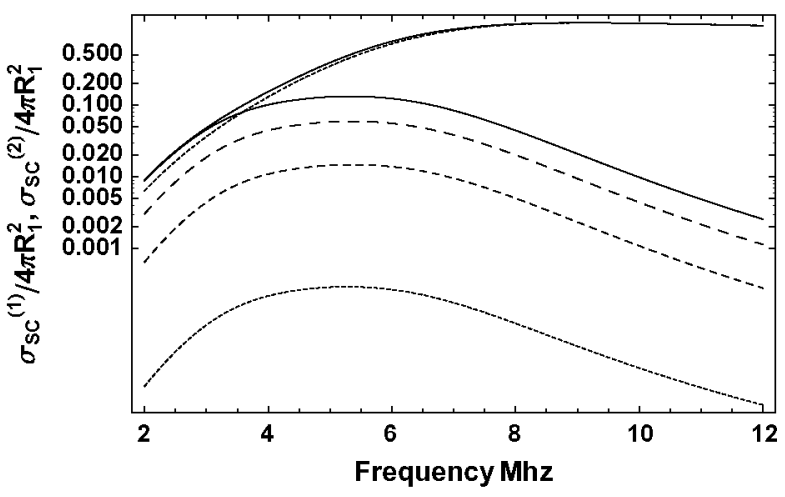

Fig. 3. (a) First and second-harmonic scattering cross-sections, $\sigma_{s c}^{(1)}$ and $\sigma_{s c}^{(2)}$ normalized with $4 \pi R_{1}^{2}$ for albumin bubbles in water as functions of the initial inner bubble radius at a transmitted frequency $f=5 \mathrm{MHz}$. Blue line correspond to $\sigma_{s 1}$ and red lines correspond to $\sigma_{s c}^{(2)}: p_{A}=30 \mathrm{kPa}$ (dotted), $p_{A}=300 \mathrm{kPa}$ (dashed), $p_{A}=500 \mathrm{kPa}$ (dark). (b) First and second-harmonic scattering cross-sections, $\sigma_{s c}^{(1)}$ and $\sigma_{s c}^{(2)}$ normalized with $4 \pi R_{1}^{2}$ for albumin bubbles in water as functions of the transmitted frequency $f$ for a initial inner bubble radius $R_{1}=2 \mu \mathrm{m}$. Blue lines correspond to $\sigma_{S 1}$ (dotted), and $\sigma_{s c}^{(1)}$ for $p_{A}=600 \mathrm{kPa}$ (dark). Red lines correspond to $\sigma_{S \mathrm{C}}^{(2)}: p_{A}=30 \mathrm{kPa}$ (dotted) $p_{A}=200 \mathrm{kPa}$ (dashed), $p_{A}=400 \mathrm{kPa}$ (long dashed), $p_{A}=600 \mathrm{kPa}$ (dark). (For interpretation of the references to colour in this figure legend, the reader is referred to the web version of this article.)

where $G$ is the shear modulus of the shell, $\mathbf{B}$ is the left Cauchy-Green tensor defined by: $\mathbf{B}=\mathbf{F F}^{T}$ and $\mathbf{F}$ is the deformation gradient tensor. The viscous component is:

$\tau_{v}^{s}=2 \eta_{s} \mathbf{D}$

where $\eta_{s}$ is the shell viscosity and $\mathbf{D}$ is the rate of strain tensor. For the rheological model described by Eqs. (11) and (12) the corresponding expression for the function $\Gamma\left(r_{1}\right)$ is:

$$
\begin{aligned}
\Gamma\left(r_{1}\right)= & -G\left\{2\left(\frac{R_{2 e}}{r_{2}}-\frac{R_{1 e}}{r_{1}}\right)+\frac{1}{2}\left[\left(\frac{R_{2 e}}{r_{2}}\right)^{4}-\left(\frac{R_{1 e}}{r_{1}}\right)^{4}\right]\right\}+4 \eta_{s} \dot{r}_{1} \\
& \times \frac{r_{1}^{3}-r_{2}^{3}}{r_{2}^{3} r_{1}}
\end{aligned}
$$

where $R_{1 e}$ and $R_{2 e}$ are the internal and external shell radii in the unstrained equilibrium state given by the roots of the equation:

$-\left(\frac{2 \sigma_{1}}{R_{1}}+\frac{2 \sigma_{2}}{R_{2}}\right)+\Gamma\left(R_{1}\right)=0$

which corresponds to the condition: $t=0: p_{0}=p_{\mathrm{g} 0}[6]$. The above system is written in dimensionless form by introducing the scales: length: $R_{1}$, time: $R_{1} \sqrt{\rho_{s} / p_{0}}$, pressure: $p_{0}$. The following nondimensional equation is obtained:

$f_{1}\left(x_{1}\right) x_{1} \ddot{x}_{1}+f_{2}\left(x_{1}\right) \dot{x}_{1}^{2}=p^{*}-p_{\infty}^{*}+\lambda M \frac{d}{d t^{*}} \frac{x_{1}\left(p^{*}-p_{\infty}^{*}\right)}{1+f\left(x_{1}\right)}$ where

$$
\begin{aligned}
& f_{1}\left(x_{1}\right)=1+f\left(x_{1}\right)-\lambda M F\left(x_{1}\right) \dot{x}_{1} \\
& f_{2}\left(x_{1}\right)=\frac{3}{2}+f\left(x_{1}\right)\left(2-\frac{x_{1}^{3}}{2 x_{2}^{3}}\right)-\frac{1}{2} \lambda M \frac{d}{d t^{*}} x_{1} F\left(x_{1}\right) \\
& f\left(x_{1}\right)=(\lambda-1) \frac{x_{1}}{x_{2}}, F\left(x_{1}\right)=\frac{1+(\lambda-1)\left(\frac{x_{1}}{x_{2}}\right)^{4}}{1+(\lambda-1) \frac{x_{1}}{x_{2}}} \\
& p^{*}\left(x_{1}\right)=\left(\frac{1}{x_{1}}\right)^{3 \gamma}-2\left(\frac{W e_{1}}{x_{1}}+\frac{W e_{2}}{x_{2}}\right)-\frac{4}{\operatorname{Re}_{1}} \frac{\dot{x}_{2}}{x_{2}}+\Gamma^{*}\left(x_{1}\right) \\
& \Gamma^{*}\left(x_{1}\right)=-G^{*}\left\{2\left(\frac{x_{2 e}}{x_{2}}-\frac{x_{1 e}}{x_{1}}\right)+\frac{1}{2}\left[\left(\frac{x_{2 e}}{x_{2}}\right)^{4}-\left(\frac{x_{1 e}}{x_{1}}\right)^{4}\right]\right\}-\frac{4}{\operatorname{Re}_{s}} \dot{x}_{1} \frac{d^{3}-1}{x_{2}^{3} x_{1}}
\end{aligned}
$$

stars, which will be suppressed hereafter, denote dimensionless quantities,

$x_{1}=r_{1} / R_{1}, x_{2}=r_{2} / R_{1}, x_{1 e}=R_{1 e} / R_{1}, x_{2 e}=R_{2 e} / R_{1}, \lambda=\frac{\rho_{l}}{\rho_{s}}$,

$d=R_{2} / R_{1}, M=\sqrt{p_{0}} / c_{s} \sqrt{\rho_{s}}$

is the Mach number, $W e_{1}=2 \sigma_{1} / R_{1} p_{0}, W e_{2}=2 \sigma_{2} / R_{1} p_{0}$ are the Weber numbers for the internal and external interfaces respectively, $\mathrm{Re}_{\mathrm{I}}=\rho_{l} R_{1} /\left(\lambda \eta_{l} \sqrt{p_{0} / \rho_{s}}\right)$ is the Reynolds number in the liquid phase, and $\operatorname{Re}_{s}=\rho_{s} R_{1} /\left(\eta_{s} \sqrt{p_{0} / \rho_{s}}\right)$ the Reynolds number in the shell.

\section{Results}

\subsection{Linear resonance frequency}

Eqs. (15)-(20) are linearized around the initial state, that is:

$x_{1}=1+z$

The perturbation $z$ satisfies the equation:

$a_{0} \ddot{z}+a_{1} \dot{z}+a_{2} z=p_{A} \sin \omega t+b \omega p_{A} \cos \omega t$

where

$a_{0}=a+b c, \quad a_{1}=b a_{2}-c$,

$a=\frac{\lambda-1}{d}+1, \quad b=\lambda M \frac{d}{d+\lambda-1}, \quad c=\frac{4}{d^{3}}\left(\frac{1}{\operatorname{Re}_{l}}+\frac{d^{3}-1}{\operatorname{Re}_{s}}\right)$

$a_{2}=-2 W e_{1}-\frac{2 W e_{2}}{d^{4}}+3 \gamma+2 G\left[x_{1 e}\left(1+x_{1 e}^{3}\right)-\frac{x_{2 e}}{d^{4}}\left(1+\frac{x_{2 e}^{3}}{d^{3}}\right)\right]$

Consider a solution of the Eq. (22) in the form:

$z(t)=\frac{B}{2} e^{i \omega t}+$ c.c.

Insertion of the above expression into Eq. (22) gives:

$B=\frac{b \omega+i}{c-a \omega^{2}+i b \omega}$

The linear resonance frequency is defined as the value of the angular frequency $\omega_{l}$ for which: $d|B| / d \omega=0$. Thus, the following expression is obtained:

$\omega_{l}=2 \pi f_{l}=\frac{1}{b}\left\{-1+\left[1-\frac{b^{2}}{a_{0}^{2}}\left(c^{2}-2 a a_{2}\right)\right]^{1 / 2}\right\}^{1 / 2}$

Note that the expression (26) is different to the one obtained by Khismatullin and Nadim [8] and Khismatullin [9] for the Kelvin-Voigt model. However, because the linear approximation corresponds to infinitesimal perturbations, numerical values obtained from the Kelvin-Voigt model or from the neo-Hookean model (Eq. 26) are nearly identical. The undamped resonance frequency is: 
(a)

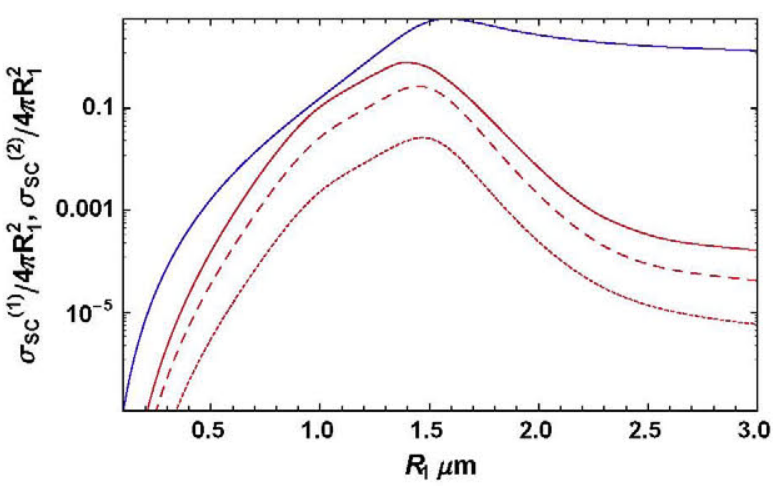

(b)

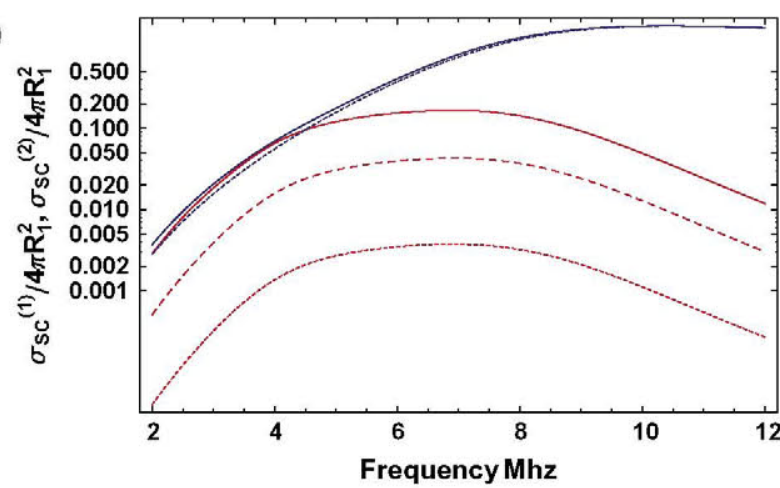

Fig. 4. (a) First and second-harmonic scattering cross-sections, $\sigma_{s c}^{(1)}$ and $\sigma_{s c}^{(2)}$ normalized with $4 \pi R_{1}^{2}$ for lipid bubbles in water as functions of the initial inner bubble radius at a transmitted frequency $f=5 \mathrm{MHz}$. Blue line correspond to $\sigma_{s 1}$ and red lines correspond to $\sigma_{s c}^{(2)}: p_{A}=30 \mathrm{kPa}$ (dotted), $p_{A}=100 \mathrm{kPa}$ (dashed), $p_{A}=200 \mathrm{kPa}$ (dark). (b) First and second-harmonic scattering cross-sections, $\sigma_{s c}^{(1)}$ and $\sigma_{s c}^{(2)}$ normalized with $4 \pi R_{1}^{2}$ for lipid bubbles in water as functions of the transmitted frequency $f$ for an initial inner bubble radius $R_{1}=1 \mu \mathrm{m}$. Blue lines correspond to $\sigma_{s 1}$ (dotted) and $\sigma_{s c}^{(1)}$ for $p_{A}=200 \mathrm{kPa}$ (dark). Red lines correspond to $\sigma_{s c}^{(2)}: p_{A}=30 \mathrm{kPa}$ (dotted), $p_{A}=100 \mathrm{kPa}$ (dashed), $p_{A}=200 \mathrm{kPa}$ (dark). (For interpretation of the references to colour in this figure legend, the reader is referred to the web version of this article.)

$$
\begin{aligned}
\omega_{0} & =2 \pi f_{0} \\
& =\left\{\frac{-2 W e_{1}-\frac{2 W e_{2}}{d^{4}}+3 \gamma+2 G\left(x_{1 e}\left(1+x_{1 e}^{3}\right)-\frac{x_{2 e}}{d^{4}}\left(1+\frac{x_{2 e}^{3}}{d^{3}}\right)\right)}{\frac{\lambda-1}{d}+1}\right\}^{1 / 2}
\end{aligned}
$$

It is straighforward to verify that if surface tension coefficients and shell viscosity are ignored and if the equilibrium radii are identified with their initial values, the above expression is reduced to the one obtained by Allen and Rashid for the neo-Hookean model [22].

\subsection{Scattering cross-sections}

The sound pressure in the liquid phase at a distance $r$ from the bubble center at $t^{\prime}=t-r / c$ may be calculated from the expression [29]:

$P_{s}(r, t)=\rho_{l} \frac{r_{2}\left(t^{\prime}\right)}{r}\left[2 \dot{r}_{2}\left(t^{\prime}\right)+r_{2}\left(t^{\prime}\right) \ddot{r}_{2}\left(t^{\prime}\right)\right]=\frac{1}{r} q_{s}\left(t^{\prime}\right)$

Expression (28) will be evaluated at the bubble surface where the time delay $r / c$ is negligible and therefore in the following, it will be considered that: $t^{\prime}=t$. The scattering cross-section $\sigma_{s c}$ is defined as the ratio of the scattered power to the intensity of the incident acoustic field [29]: $\sigma_{s c}=4 \pi \frac{\left\langle q_{s}^{2}(t)\right\rangle_{t}}{\left\langle p_{\Lambda}^{2}(t)\right\rangle_{t}}$

where $\langle 0\rangle_{t}$ denotes a mean time:

$\left\langle q_{s}^{2}(t)\right\rangle_{t}=\frac{\int_{\tau_{1}}^{\tau_{2}} q_{s}^{2}(t) d t}{\tau_{2}-\tau_{1}}$

$\left\langle p_{A}^{2}(t)\right\rangle_{t}=\frac{\int_{\tau_{1}}^{\tau_{2}} p_{A}^{2}(t) d t}{\tau_{2}-\tau_{1}}$

and $\tau_{2}-\tau_{1}$ is a time interval after the initial transients have elapsed.

For infinitesimal acoustic pressure amplitudes, the first-harmonic scattering cross-section denoted $\sigma_{s 1}$ may be obtained by introducing in the above equations the linear approximation (24). This procedure leads to the following analytical expression:

$\sigma_{s 1}=4 \pi R_{1}^{2}\left(\lambda^{2} \omega^{4}|B|^{2}\right)$

where the amplitude $B(\omega)$ is given by the expression (25).

For finite pressure amplitudes the scattering cross-sections $\sigma_{s c}$ has been determined by means of the numerical integration of the system (15)-(20) for 120 acoustic cycles, and ulterior numerical evaluation of expression (29) in the interval 100-120, that is, once transient effects have been died out (all calculations have been performed with Mathematica ${ }^{(B)}$ ). Results will be obtained by considering, for the shell properties and the rest of physical parameters involved in the problem, experimental data provided in previous works in the literature. Thus, for albumin-shelled microbubbles in water: $\rho_{s}=1100 \mathrm{~kg} / \mathrm{m}^{3}, G=88.8 \mathrm{MPa}, \eta_{s}=1.77 \mathrm{~Pa} \mathrm{~s}, \sigma_{1}=0.04 \mathrm{~N} / \mathrm{m}$, $\sigma_{2}=0.005 \mathrm{~N} / \mathrm{m}, \rho_{l}=1000 \mathrm{~kg} / \mathrm{m}^{3}, \eta_{l}=0.001$ Pa s, $c_{s}=1450 \mathrm{~m} / \mathrm{s}, \gamma=$ 1.4 , layer thickness $R_{2}-R_{1}=15 \mathrm{~nm}$ (data from $[6,30]$ ); for polymeric bubbles in blood: $\rho_{s}=1100 \mathrm{~kg} / \mathrm{m}^{3}, G=12 \mathrm{MPa}, \eta_{s}=0.45 \mathrm{~Pa} \mathrm{~s}$, $\sigma_{1}=0.04 \mathrm{~N} / \mathrm{m}, \sigma_{2}=0.005 \mathrm{~N} / \mathrm{m}, \rho_{l}=1027 \mathrm{~kg} / \mathrm{m}^{3}, \eta_{l}=0.0012$ Pa s, $c_{s}=1543 \mathrm{~m} / \mathrm{s}, \gamma=1.1$, layer thickness $15 \%$ of the initial outer radius (data from [7,9]); for lipid-shelled bubbles in water: $\rho_{s}=1100 \mathrm{~kg} /$ $\mathrm{m}^{3}, G=50 \mathrm{MPa}, \eta_{\mathrm{s}}=0.8 \mathrm{~Pa} \mathrm{~s}, \sigma_{1}=0.04 \mathrm{~N} / \mathrm{m}, \sigma_{2}=0.005 \mathrm{~N} / \mathrm{m}, \gamma=$ 1.1 , layer thickness $R_{2}-R_{1}=4 \mathrm{~nm}$ (data from [30]).

Numerical results for $\sigma_{s c}$ normalized by $4 \pi R_{1}^{2}$ as function of the transmitted frequency normalized by the linear resonance frequency $f_{l}$ for increasing pressure amplitudes are shown in Fig. 1. In Fig. $1 \mathrm{a}$ the initial inner bubble radius is $R_{1}=3 \mu \mathrm{m}$, and the pressure amplitudes $p_{A}=300,500,700 \mathrm{kPa}$, respectively; in Fig. $1 \mathrm{~b}: R_{1}=4 \mu \mathrm{m}$, and $p_{A}=500,700,1000 \mathrm{kPa}$; and finally in Fig. $1 \mathrm{c}: R_{1}=1.5 \mu \mathrm{m}$, and $p_{A}=100,200,300 \mathrm{kPa}$. In the three graphics, the curves corresponding to $\sigma_{s 1}$ have been also included.

These plots show a clear increase of the scattering cross-sections for growing pressure amplitudes. However, the quantitative divergences of $\sigma_{s c}$ with respect to the linear first-harmonic scattering cross-section $\sigma_{s 1}$ obtained from Eq. (32), are not so important as those reported for free-bubbles where this increase may be even of several orders of magnitude [29]. In fact, for rigid encapsulations (albumin and polymer microbubbles) appreciable increments are only observed for large size bubbles. Indeed, the results obtained show that for albumin bubbles of a initial radius $R_{1}=3 \mu \mathrm{m}$, the normalized scattering cross-section increases from 3.79 (linear value) to 5.34 for an acoustic pressure $p_{A}=700$ that is, an increase of about $40 \%$. For polymeric bubbles of a radius $R_{1}=4 \mu \mathrm{m}$ (twice the mean radius of a polydisperse solution) the increase is only of $20 \%$ when the acoustic pressure grows to $p_{A}=1 \mathrm{MPa}$. The deviation from the linear values is, however, very significant for the more flexible lipid-shelled microbubbles (Fig. 1c). Indeed, for a initial bubble radius $R_{1}=1.5 \mu \mathrm{m}$ (mean radius), $\sigma_{s c}$ experiences an increasing of $70 \%$ at a moderately large acoustic pressure amplitude $p_{A}=300 \mathrm{kPa}$.

As the acoustic pressure increases, the sound emission is distributed between the different harmonics that, as it has been 
(a)

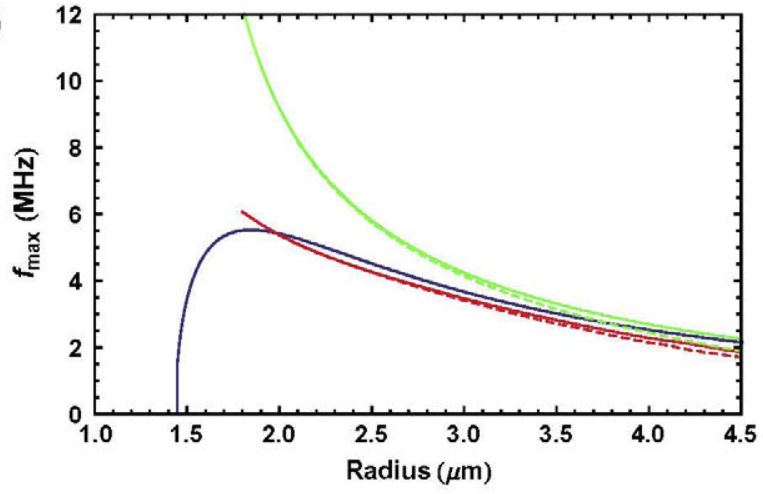

(b)

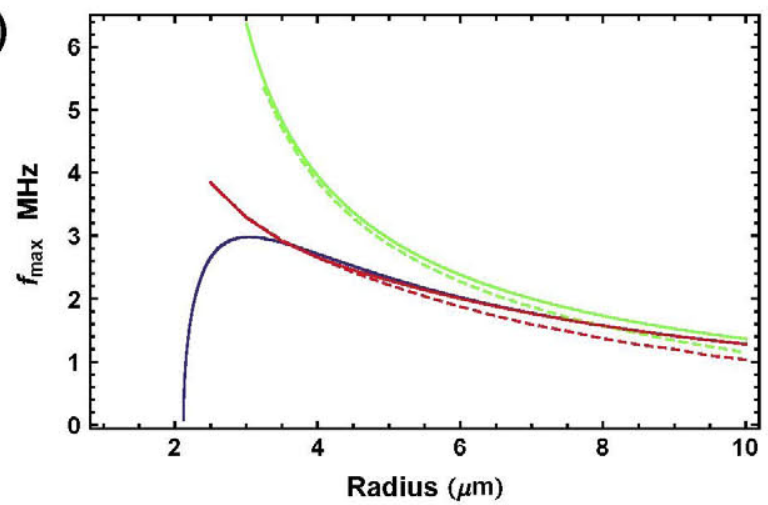

(c)

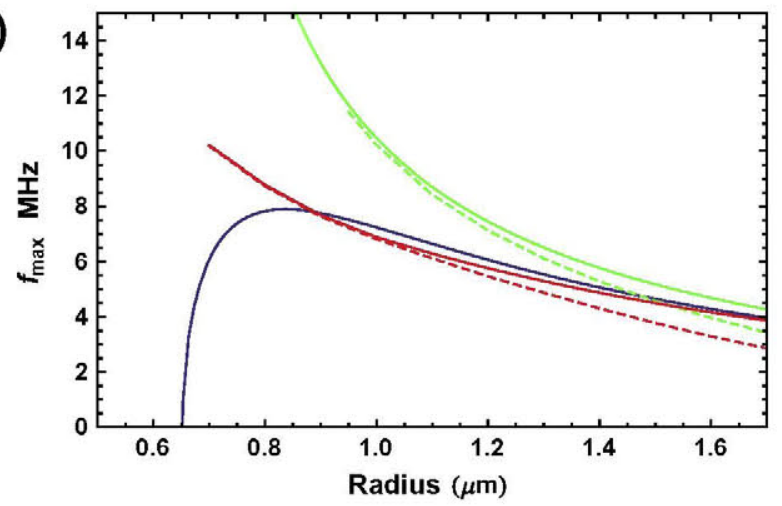

Fig. 5. First and second harmonic resonance frequencies versus initial inner bubble radius for: (a) albumin-shelled bubbles in water: blue line: linear resonance $f_{l}$, green dark line: frequency for maximum $\sigma_{s 1}$, green dashed line: $f_{s c}^{(1)}$ for $p_{A}=400 \mathrm{kPa}$. Red dark line: $f_{s c}^{(2)}$ for $p_{A}=30 \mathrm{kPa}$ : red dashed line: $f_{s c}^{(2)}$ for $p_{A}=400 \mathrm{kPa}$. (b) Polymershelled bubbles in blood: blue line: linear resonance $f_{l}$, green dark line: frequency for maximum $\sigma_{s 1}$. green dashed line: $f_{s c}^{(1)}$ for $p_{A}=700 \mathrm{kPa}$. Red dark line: $f_{s c}^{(2)}$ for $p_{A}=30 \mathrm{kPa}$ : red dashed line: $f_{s c}^{(2)}$ for $p_{A}=700 \mathrm{kPa}$. (c) Lipid-shelled bubbles in water: blue line: linear resonance $f_{b}$ green dark line: frequency for maximum $\sigma_{s 1}$, green dashed line: $f_{s c}^{(1)}$ for $p_{A}=300 \mathrm{kPa}$. Red dark line: $f_{s c}^{(2)}$ for $p_{A}=30 \mathrm{kPa}$ : red dashed line: $f_{s c}^{(2)}$ for $p_{A}=300 \mathrm{kPa}$. (For interpretation of the references to colour in this figure legend, the reader is referred to the web version of this article.)

previously discussed, must be analyzed separately. To this end, the scattering cross-sections defined by (29) will be numerically calculated in the frequency domain by means of the Fourier transform algorithms provide by the package Mathematica. The quantitative influence of the pressure amplitude on the first and second harmonic scattering cross-sections, denoted here by $\sigma_{s c}^{(1)}$ and $\sigma_{s c}^{(2)}$ respectively, are shown in Figs. 2-4. For polymeric bubbles, both sections $\sigma_{s c}^{(1)}$ and $\sigma_{s c}^{(2)}$, normalized with $4 \pi R_{1}^{2}$ are plotted for different pressure amplitudes as functions of the initial inner bubble radius for a transmitted frequency $f=5 \mathrm{MHz}$ in Fig. 2a; and as functions of the transmitted frequency for an initial bubble radius $R_{1}=2 \mu \mathrm{m}$ (a)

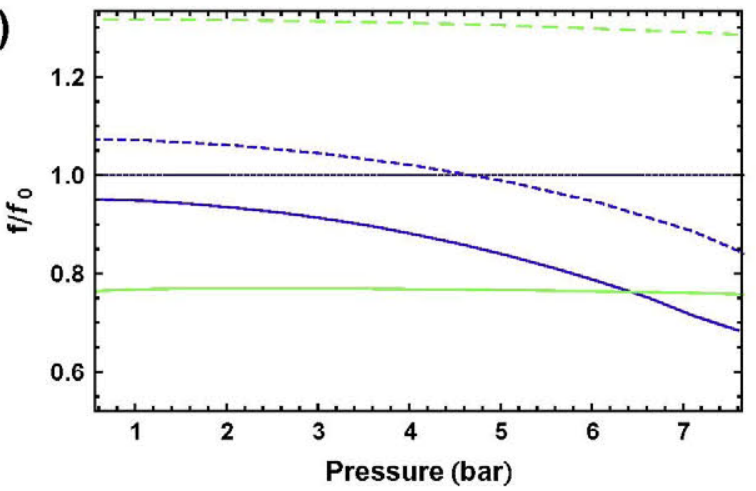

(b)

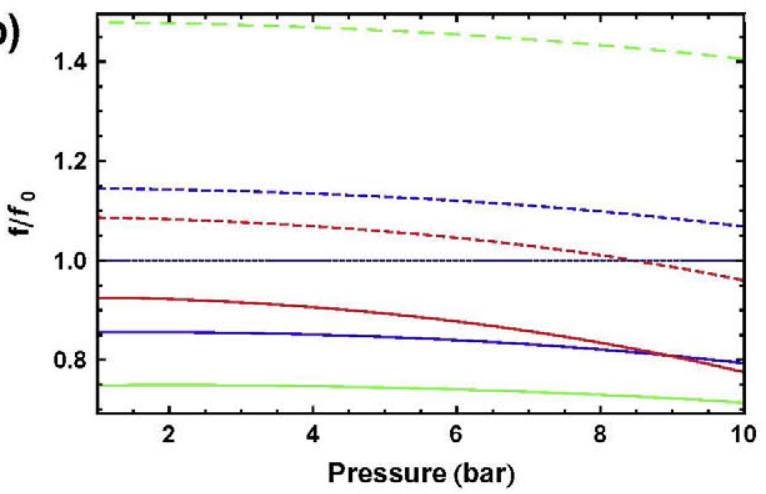

(c)

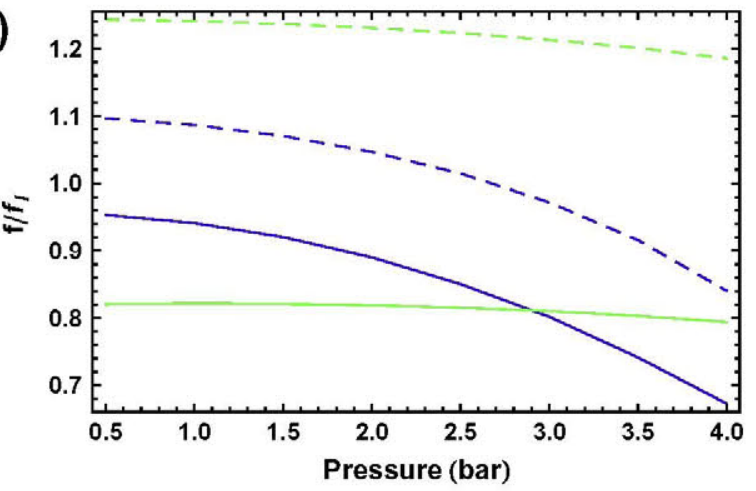

Fig. 6. First and second harmonic resonance frequencies normalyzed by the undamped resonance frequency $f_{0}$ as function of the pressure amplitude for: (a) albumin-shelled bubbles in water: green lines: $R_{1}=2 \mu \mathrm{m}$, blue lines: $R_{1}=3.5 \mu \mathrm{m}$. dashed lines: $f_{s c}^{(1)}$, dark lines: $f_{s c}^{(2)}$. (b) Polymer-shelled bubbles in blood green lines: $R_{1}=3 \mu \mathrm{m}$, blue lines: $R_{1}=5 \mu \mathrm{m}$, red lines $R_{1}=7 \mu \mathrm{m}$, dashed lines: $f_{s c}^{(1)}$, dark lines: $f_{s c}^{(2)}$. (c) Lipid-shelled bubbles in water: green lines: $R_{1}=1 \mu \mathrm{m}$, blue lines: $R_{1}=1.5 \mu \mathrm{m}$. dashed lines: $f_{s c}^{(1)}$, dark lines: $f_{s c}^{(2)}$. (For interpretation of the references to colour in this figure legend, the reader is referred to the web version of this article.)

in Fig. 2b. The above values of $f$ and $R_{1}$ have been chosen in order to accomplish a direct comparison with the previous results provided by Khismatullin [9] for the Kelvin-Voigt model in the linear domain. As expected, the results here obtained for the neo-Hookean model are very close to those provided in [9] for both, the linear first harmonic scattering cross-section $\sigma_{s 1}$ and the second harmonic scattering cross-section for a small pressure amplitude: $p_{A}=30 \mathrm{kPa}$. As it may be observed in Fig. $2 \mathrm{a}$ and b, $\sigma_{s c}^{(1)}$ is nearly unaffected by $p_{A}$ (in fact, its influence is so small that cannot be diferenciated in the plots) whereas the second-harmonic $\sigma_{s c}^{(2)}$ increases dramatically as the pressure amplitude growths. This result may be of special relevance for second harmonic imaging and it will be discussed later. It must be also noted in Fig. $2 b$ that, as it was pointed out in [9], for 


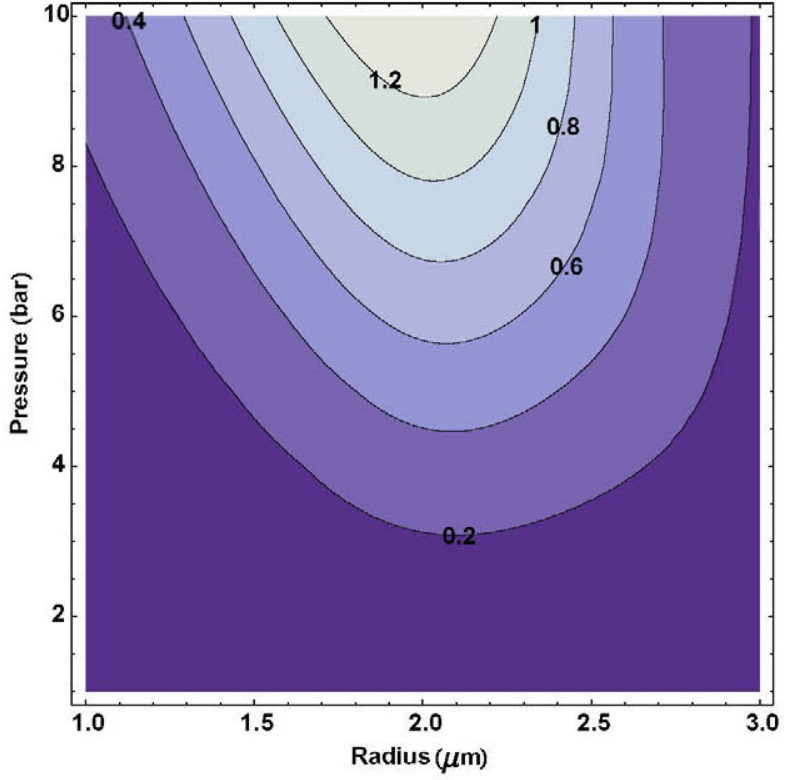

Fig. 7. Contour plot of the ratio of second to first harmonic scattering cross-sections as function of the initial inner bubble radius $R_{1}$ and the pressure amplitude $p_{A}$ for albumin-shelled bubbles in water at a driven frequency $f=4 \mathrm{MHz}$.

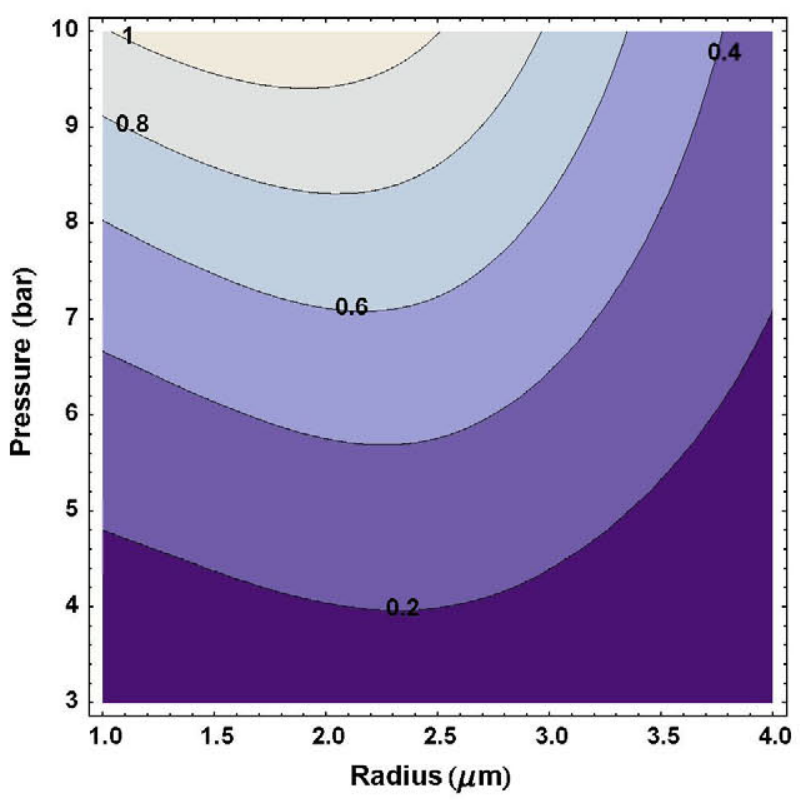

Fig. 8. Contour plot of the ratio of second to first harmonic scattering cross-sections as function of the initial inner bubble radius $R_{1}$ and the pressure amplitude $p_{A}$ for polymer-shelled bubbles in blood at a driven frequency $f=3 \mathrm{MHz}$.

$R_{1}=2 \mu \mathrm{m}$, and for any pressure amplitude $p_{A}$, the sections $\sigma_{s c}^{(2)}$ reach a local maximum for some frequency $f$, whereas there is no value of the transmitted frequency which maximize $\sigma_{s c}^{(1)}$, on the contrary, they tend to asymptotic values as $f$ increases. This is due to the existence of a critical radius below which no first harmonic resonance frequency exists, as it was shown in [9] for infinitesimal acoustic pressures. In the present analysis it has been found for $\sigma_{1 s}$ (linear approximation) a critical value $R_{1 c}=2.43 \mu \mathrm{m}$. It has been also verified that the critical radius values corresponding to $\sigma_{1 s}$ are nearly unaltered by finite pressure amplitudes. If the existence of this critical radius in the linear domain suggests the possibility of second harmonic imaging in suspensions of polymeric bubbles with a mean radius $2-2.5 \mu \mathrm{m}$, for a transmitted frequency around $f=5 \mathrm{MHz}$ [9], the results here obtained (Fig. 2b) confirm this prediction and show that this possibility should be enhanced for large acoustic pressure amplitudes.

The sections $\sigma_{s c}^{(1)}$ and $\sigma_{s c}^{(2)}$, normalized with $4 \pi R_{1}^{2}$ for albumin coated microbubbles in water are plotted for different pressure amplitudes as functions of the initial inner bubble radius for a transmitted frequency $f=5 \mathrm{MHz}$ in Fig. 3a; and as functions of the transmitted frequency for an initial bubble radius $R_{1}=2 \mu \mathrm{m}$ in Fig. 3b. The behavior shown in these plots is similar to the one described above for polymeric bubbles. The section $\sigma_{s c}^{(1)}$ is unaltered by the driving pressure $p_{A}$, however, as it may be observed, $\sigma_{s c}^{(2)}$ experiences an increment of several order of magnitude when the pressure amplitude grows from $p_{A}=30 \mathrm{kPa}$ to $p_{A}=500 \mathrm{kPa}$.

For lipid shelled microbubbles the strong increase predicted for the total $\sigma_{s c}$ is transmitted to the harmonic content even for bubbles of small size. Results for lipid bubbles in water for $\sigma_{s c}^{(1)}$ and $\sigma_{s c}^{(2)}$ as functions of the initial inner bubble radius for a transmitted frequency $f=5 \mathrm{MHz}$ are plotted in Fig. 4a; and as functions of the transmitted frequency for an initial bubble radius $R_{1}=1 \mu \mathrm{m}$ in Fig. $4 \mathrm{~b}$. The behavior observed here for low pressure amplitudes $p_{A} \approx 30-200 \mathrm{kPa}$, is very similar to the one described previously for albumin or polymeric shelled bubbles (Fig. 3a and b) for larger amplitudes with a significant increase of the second harmonic scattering cross-section $\sigma_{s c}^{(2)}$. In fact, $\sigma_{s c}^{(2)}$ exceeds $\sigma_{s c}^{(1)}$ for pressures above $p_{A}=200 \mathrm{kPa}$. Note also that for this class of encapsulation first harmonic resonance frequencies are found except for very tiny microbubbles, since the critical radius is $R_{1 c}=0.71 \mu \mathrm{m}$.

\subsection{Nonlinear harmonic resonance frequencies}

As pointed out by Khismatullin and Nadim [8] and Khismatullin [9], every harmonic of the backscatter signal has its own resonance frequency. This is confirmed in the curves shown in Figs. 2-4 where as it has been previously discussed, $\sigma_{s c}^{(2)}$ and $\sigma_{s c}^{(1)}$ (if $R_{1}>R_{1 c}$ ) have a local maximum for given values of the transmitted frequency for each value of the acoustic pressure amplitude. The transmitted frequencies at wich these resonance peaks of the first and second scattering cross-sections versus frequency occur, will be identified in the following as the (nonlinear) first and second harmonic resonances frequencies. The explicit dependence of these frequencies, hereafter denoted $f_{s c}^{(1)}$ and $f_{s c}^{(2)}$, on the initial inner bubble radius for different pressure amplitudes is illustrated in Fig. 5 for albumin, polymer and lipid encapsulated bubbles respectively. The corresponding linear resonance curves resulting from Eq. (26), have been also included. In Fig. 5 a the pressure amplitudes considered are: $p_{A}=30,400 \mathrm{kPa}$, respectively, in Fig. $5 \mathrm{~b}: p_{A}=30,700 \mathrm{kPa}$, and in Fig. $5 \mathrm{c}: p_{A}=30,300 \mathrm{kPa}$.

For the three encapsulations it is observed that the divergences relative to the linear values increases as the initial bubble radius increases. However, very different behavior is detected between hard encapsulations (albumin and polymer bubbles) and soft encapsulation (lipid-shelled bubbles). For the first group it must be noted in Fig. $5 a$ and $b$, that for small bubbles the influence of the driving pressure amplitude on both first and second harmonic resonance frequencies, is very small. According to the results obtained, it may be concluded that for albumin and polymer encapsulated bubbles of initial bubbles radius about the mean radius of a polydisperse solution $(1.7 \mu \mathrm{m}$ for albumin-shelled bubbles, $2.0-2.5 \mu \mathrm{m}$ for polymer shelled bubbles) or smaller, the values corresponding to analytical expressions obtained from linear approaches like expression (32) or expressions (17a) and (17b) in Ref. [8], provided a good approximation to determine harmonic resonance frequencies. For bubble radii slightly larger than the mean value, the divergences are small but not negligible tacking into account the high sensitivity of modern contrast imaging 


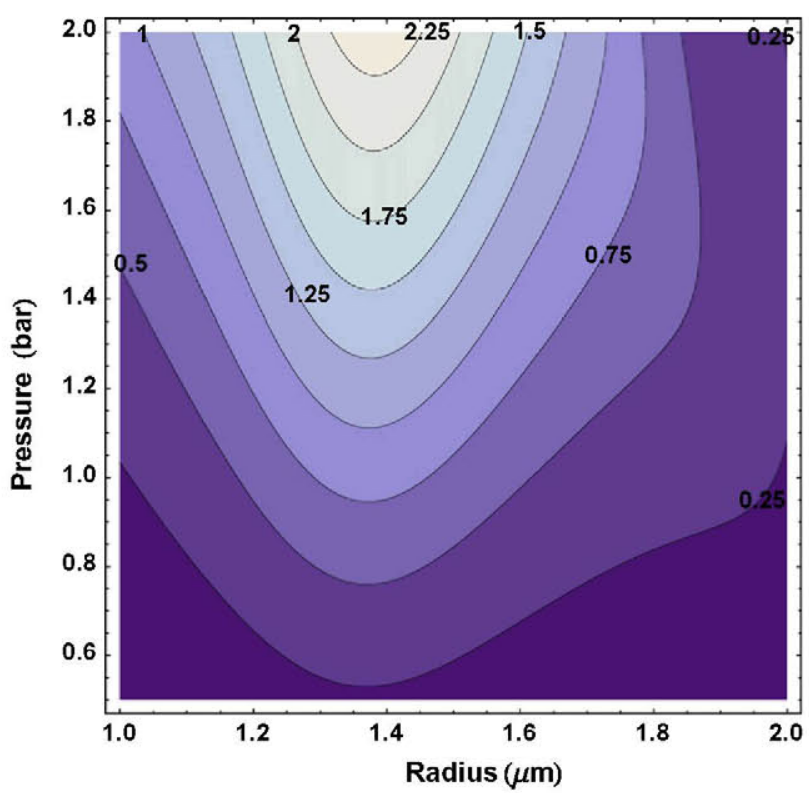

Fig. 9. Contour plot of the ratio of second to first harmonic scattering cross-sections as function of the initial inner bubble radius $R_{1}$ and the pressure amplitude $p_{A}$ for lipid-shelled bubbles in water at a driven frequency $f=3 \mathrm{MHz}$.

equipment. The discrepancies between linear and nonlinear values are however significant for lipid bubbles except in the submicron region (Fig. 5c).

The harmonic resonance frequencies are shifted to smaller values as the acoustic pressure increases as it may be seen in Fig. 6 where this explicit quantitative dependence is shown. In Fig. 6a results are plotted for albumin bubbles and for initial inner bubble radii $R_{1}=2,3.5 \mu \mathrm{m}$. In Fig. $6 \mathrm{~b}$ for polymeric bubbles and $R_{1}=3,5$, $7 \mu \mathrm{m}$, and in Fig. $6 \mathrm{c}$ for lipid bubbles and $R_{1}=1,1.5 \mu \mathrm{m}$. As noted previously, for albumin and polymeric bubbles of a radius of the order of the mean radius, first and second frequencies are nearly invariant for pressures below $500 \mathrm{kPa}$. For albumin bubbles of a radius $R_{1}=3.5 \mu \mathrm{m}$, the harmonic resonance $f_{s c}^{(1)}$ experiences a reduction of about $17 \%$ and $f_{s c}^{(2)}$ of the order of $25 \%$,when the acoustic pressure changes from $100 \mathrm{kPa}$ to $700 \mathrm{kPa}$. For polymeric bubbles the reduction is negligible for small bubble sizes and moderate for large bubbles, for instance, is lesser than $10 \%$ for bubbles with $R_{1}=5 \mu \mathrm{m}$ even if the pressure is increased to $1 \mathrm{MPa}$.The situation is very different for lipid encapsulated bubbles. In this case, the reduction is of the order of $23 \%$ for $f_{s c}^{(1)}$ and of the order of $30 \%$ for $f_{s c}^{(2)}$ for bubbles of the mean radius $R_{1}=1.5 \mu \mathrm{m}$ when the pressure has a moderate increase: from $50 \mathrm{kPa}$ to $400 \mathrm{kPa}$.

\subsection{Second harmonic imaging}

The above results provide important information about transmitted resonance frequency values at which a high intensity of the second harmonic scattered signal may be expected. However, as noted by Grossmann et al. [34] second harmonic imaging relies on the contrast between fundamental and second harmonic intensities, thus, in order to optimize second harmonic imaging the ratio of second harmonic intensity to fundamental must be maximized. According to the results shown in the above sections, increasing of the pressure amplitude enhances the intensity of the second harmonic and therefore, insonification with fields of large acoustic amplitude should be a sufficient condition to fulfil the above requirement. However, the pressure amplitudes must be bounded. First, for safety considerations, the Mechanical Index must be restricted to small values. Second, similarly to free bubbles, above a certain threshold of the acoustic amplitude parametric instability appears, as it has been recently detected in experiments [31,32] and predicted theoretically [33]. Indeed, for a particular class of lipid-coated microbubbles of radii between 2 and $4 \mu \mathrm{m}$, at a frequency $1.7 \mathrm{MHz}$, an acoustic pressure of $100 \mathrm{kPa}$ is sufficient to excite surface modes, and shell rupture occurs for small bubbles at pressure above $200 \mathrm{kPa}$ [31]. At present, however, there is no experimental data available at each frequency for the different types of encapsulation. Consequently, in this study calculations has been performed for acoustic pressures sufficient to illustrate the more relevant aspects associated to the nonlinear behavior, but small enough to provide moderate values of the Mechanical Index. Thus, the maximum value considered for lipid-shelled bubbles is $M I \approx 0.18$, for albumin-shelled bubbles $M I \approx 0.5$, and for polymer-shelled bubbles $M I \approx 0.58$. The task is, therefore, to determine the parameters region where the ratio $\sigma_{s c}^{(2)} / \sigma_{s c}^{(1)}$ is maximized with minimum ultrasound amplitudes. Similar analyses have been previously performed for free-bubbles by Grossmann et al. [34] and by Mukdadi et al. [35] for bubbles encapsulated by a layer of a incompressible newtonian fluid.

The results here obtained are shown in the contour plots included in Figs. 7-9. The ratio $\sigma_{s c}^{(2)} / \sigma_{s c}^{(1)}$ is plotted versus initial radius $R_{1}$ and pressure amplitude $p_{A}$ at a fixed driving frequency $f=4 \mathrm{MHz}$ for albumin microbubbles in Fig. 7, for polymer microbubbles at $f=3 \mathrm{MHz}$ in Fig. 8 and for lipid microbubbles at $f=3 \mathrm{MHz}$ in Fig. 9, respectively. As it can be observed in Fig. 7, for albumin-shelled microbubbles at $f=4 \mathrm{MHz}$ optimal conditions correspond to bubble sizes in the range: $R_{1}=1.5-2.5 \mu \mathrm{m}$. Furthermore, $\sigma_{s c}^{(2)}$ exceeds $\sigma_{s c}^{(1)}$ for $R_{1}=2 \mu \mathrm{m}$ at pressure amplitudes above $800 \mathrm{kPa}$. For this bubble radius and this pressure amplitude, the second harmonic resonance frequency is $f_{s c}^{(2)}=5.28 \mathrm{MHz}$. For polymer-shelled microbubbles high values of $\sigma_{s c}^{(2)} / \sigma_{s c}^{(1)}$ at $f=3 \mathrm{MHz}$ may be found for $R_{1}=1-3 \mu \mathrm{m}$ and, therefore, for $R_{1}$-values in the interval of the mean radius of polydisperse solutions: $R_{1}=2-2.5 \mu \mathrm{m}$. Note, however, that the pressure amplitudes needed to reach a quotient $\sigma_{s c}^{(2)} / \sigma_{s c}^{(1)}$ larger than unity are very high, in particular, for $R_{1}=2.5 \mu \mathrm{m}$, acoustic amplitude values above $900 \mathrm{kPa}$ are required. For $R_{1}=2.5 \mu \mathrm{m}$, high values of $\sigma_{s c}^{(2)} / \sigma_{s c}^{(1)}$ are observed for the transmitted frequency $f=3 \mathrm{MHz}$ which is smaller than those corresponding to second harmonic resonance frequency; for instance, $f_{s c}^{(2)}=3.83 \mathrm{MHz}$ for $p_{A}=700 \mathrm{kPa}$. For lipid-shelled bubbles optimal bubble radii at $f=3 \mathrm{MHz}$ are found in the interval $R_{1}=1.2-1.6 \mu \mathrm{m}$. For $R_{1}=1.6 \mu \mathrm{m}, \sigma_{s c}^{(2)}$ exceeds $\sigma_{s c}^{(1)}$ for pressure amplitudes above $110 \mathrm{kPa}$, and the second harmonic resonance frequency for $p_{A}=300 \mathrm{kPa}$ is $f_{s c}^{(2)}=3.29 \mathrm{MHz}$. In principle, $\sigma_{s c}^{(2)}$ could exceed $\sigma_{s c}^{(1)}$ for smaller pressure amplitudes than those quoted above at low frequencies (far from resonance) as suggest the plots in Figs. 3b and 4b. Note, however that at this frequencies both $\sigma_{s c}^{(2)}$ and $\sigma_{s c}^{(1)}$ may have extremely low values, i.e. probably insufficient to overcome the noise level.

The above results suggest that high contrast between second harmonic intensity and fundamental intensity for moderate acoustic amplitudes may be expected for mean values of the bubble radius of polydisperse solutions at transmitted frequencies smaller and relatively close to those corresponding to the second harmonic resonance frequency and, therefore, smaller than the linear resonance frequency as well as the first harmonic resonance frequency. These conditions for second harmonic imaging could be optimized if the dispersion in microbubble size is reduced. Recent experimental findings about monodisperse solutions of contrast agent microbbules [36] may contribute notably on this ultrasound application.

\section{Concluding remarks}

In this paper a theoretical analysis of the scattering of encapsulated bubbles immersed in a liquid and irradiated by ultrasound 
fields of large acoustic pressure amplitudes has been performed. The equations for radial bubble oscillations in a compressible fluid have been derived by considering, for the viscoelastic shell, an isotropic hyperelastic neo-Hookean model for the elastic contribution in addition to a Newtonian viscous component. From a linear analysis of the governing equations, valid for infinitesimal driving pressure amplitudes, analytical expressions for the resonance frequency as well as for the (linear) first harmonic scattering cross-section have been determined. For finite amplitudes, i.e. in the nonlinear regime, emphasis has been focused on analyzing the harmonic content of the backscatter signal. Consequently, the quantitative influence of the driving pressure on the harmonic scattering cross-sections and harmonic resonance frequencies has been investigated for different equilibrium bubble sizes. Numerical results have been obtained for albumin, polymer and lipid encapsulating layers, and may be summarized as follows:

Harmonic scattering cross-sections growth as the pressure amplitudes are increased. For typical values of the mean radius of polydisperse solutions, the quantitative influence of the driving pressure on the first harmonic scattering cross-section is negligible, whereas the second harmonic scattering cross-section increases dramatically with increasing pressure amplitude.

For albumin and polymeric encapsulated bubbles of initial equilibrium radii around the respective mean value, harmonic resonance frequencies are nearly unaffected by the pressure driving amplitude. Thus, the analytical expressions for harmonic resonance frequencies obtained from a linear approach provide a good approximation even for moderately large pressure amplitudes. For lipid-shelled microbubbles, significant discrepancies between linear and nonlinear values have been detected and, therefore, to determine harmonic resonance frequencies a numerical analysis like the one here performed should be carried out.

Conditions for optimal second harmonic imaging have been also investigated and some regions in the parameters space where the second harmonic intensity is dominant over the fundamental have been identified. The results obtained suggest that a high contrast between second harmonic intensity and fundamental intensity for moderate acoustic amplitudes may be expected for mean values of the bubble radius of polydisperse solutions at transmitted frequencies slightly smaller than those corresponding to the second harmonic resonance frequency.

Second harmonic imaging is a useful technique that has been largely used. However, it has some limitations because the harmonics generated may be reflected by the surrounding tissue with the subsequent reduction of resolution. To overcome these restrictions the use of harmonics of higher order [37], or subharmonic ultrasound signals has been proposed [38,39]. Consequently, the analysis given here could be extended in order to determine the range of governing parameters where subharmonic and superharmonic imaging are optimized. On the other hand, it must be stressed that the isotropic hiperelastic model may not be an adequate rheological model to describe some responses of contrast agents experimentally observed [15-17], in particular, shell viscoelastic properties may change with bubble size [14]. Thus, it may be of interest to carry out similar analysis to the one here performed by considering other nonlinear constitutive equations [40].

\section{Acknowledgements}

Fruitful remarks from Dr. J. Carpio on frequency domain calculations are thankfully appreciated. The author would also like to thank the anonimous reviewer for his/her useful comments.

\section{References}

[1] B.B. Goldberg, J.S. Raichlen, F. Forsberg (Eds.), Ultrasound Contrast Agents. Basic Principles and Clinical Applications. Martin Dunitz, 2001.

[2] V. Sboros, Response of contrast agents to ultrasound, Adv. Drug Deliv. Rev. 60 (2008) 1117-1136.

[3] K. Ferrara, R. Pollard, M. Borden, Ultrasound microbubble contrast agents: fundamentals and application to gene and drug delivery, Ann. Rev. Biomed. Eng. 9 (2007) 415-447.

[4] N. de Jong, R. Cornet, C.T. Lancée, Higher harmonics of vibrating gas-filled microspheres. Part one: simulations, Ultrasonics 32 (1994) 447-453.

[5] P.J.A. Frinking, N. de Jong, Acoustic modeling of shell encapsulated gas bubble, Ultrasound Med. Biol. 24 (1998) 523-553.

[6] Ch.C. Church, The effects of an elastic solid surface layer on the radial pulsations of gas bubbles, J. Acoust. Soc. Am. 97 (1995) 1510-1521.

[7] L. Hoff, P.C. Sontum, J.M. Hovem, Oscillation of polymeric microbubbles: effect of the encapsulating shell, J. Acoust. Soc. Am. 107 (2000) 2272.

[8] D.B. Khismatullin, A. Nadim, Radial oscillations of encapsulated microbubbles in viscoelastic liquids, Phys. Fluids 14 (10) (2002) 3534-3557.

[9] D.B. Khismatullin, Resonance frequency of microbubbles: effect of viscosity, J. Acoust. Soc. Am. 116 (2004) 1463-1473.

[10] D. Chatterjee, K. Sarkar, A newtonian rheological model for the interface of microbubble contrast agents, Ultrasound Med. Biol. 29 (2003) 1749-1757.

[11] K. Sarkar, W.T. Shi, D. Chatterjee, F. Fosberg, Characterization of ultrasound contrast microbubbles using in vitro experiments and viscous and viscoelastic interface models for encapsulation, J. Acoust. Soc. Am. 118 (2005) 539-550.

[12] S.P. Paul, A. Katiyar, K. Sarkar, D. Chatterjee, W.T. Shi, F. Forsberg, Material characterization of the encapsulation of an ultrasound contrast microbubble and its subharmonic response: strain-softening interfacial elasticity model, J. Acoust. Soc. Am. 127 (2010) 3846-3857.

[13] E. Stride, The influence of surface adsorption on microbubble dynamics, Philos. Trans. Roy. Soc. A 366 (2008) 2103-2115.

[14] S.M. Van der Meer, B. Dollet, M.M. Voormolen, Chien T. Chin, A. Bouakaz, N. de Jong, M. Versluis, D. Lohse, Microbubble spectroscopy of ultrasound contrast agents, J. Acoust. Soc. Am. 121 (1) (2007) 648-656.

[15] M. Overvelde, V. Garbin, J. Sijl, B. Dollet, N. de Jong, D. Lohse, M. Verluis, Nonlinear shell behavior of phospholipid-coated microbubbles, Ultrasound Med. Biol. 36 (2010) 2080-2092.

[16] P. Marmottant, S. van der Meer, M. Emmer, M. Versluis, N. de Jong, S. Hilgenfeld, D. Lohse, A model for large amplitude oscillations of coated bubbles accounting for buckling and rupture, J. Acoust. Soc. Am. 118 (2005) 3499-3505.

[17] N. de Jong, M. Emmer, C.T. Chin, A. Bouakaz, F. Mastik, D. Lohse, M. Versluis, "Compression-only" behavior of phospholipid-coated contrast bubbles, Ultrasound Med. Biol. 33 (2007) 653-656.

[18] C.A. MacDonald, V. Sboros, J. Gomatam, S.D. Pye, C.M. Moran, W.N. McDicken, A numerical investigation of the resonance of gas filled micrbubbles: dependence on acoustic pressure amplitude, Ultrasonics 43 (2004) 113-122.

[19] K.E. Morgan, J.S. Allen, P.A. Dayton, J.E. Chomas, A.L. Kibanov, K.W. Ferrara, Experimental and theoretical evaluation of microbubble behavior: effect of transmitted phase and bubble size, IEEE Trans. Ultrason. Ferroelect. Freq. Control 47 (6) (2000) 1494-1509.

[20] J. Wu, J. Pepe, W. Dewitt, Nonlinear behaviors of contrast agents relevant to diagnostic and therapeutic application, Ultrasound Med. Biol. 29 (2003) 555 562.

21] A.A. Doinikov, J.F. Haac, P.A. Dayton, Resonance frequencies of lipid-shelled microbubbles in the regime of nonlinear oscillations, Ultrasonics 40 (2009) 263-268.

[22] J.S. Allen, M.M. Rashid, Dynamics of a hyperelastic gas-filled spherical shell in a viscous fluid, Journal of Applied Mechanics 71 (2004) 195-200.

[23] J. Jiménez-Fernández, Dynamics of an acoustically driven encapsulated gas bubble immersed in a liquid, in: Proceeding ICA 2007, September 2007, Madrid, Spain.

[24] K. Tsiglifis, N.A. Pelekasis, Nonlinear radial oscillations of encapsulated microbubbles subject to ultrasound: the effect of membrane constitutive law, J. Acoust. Soc. Am. 123 (2008) 4059-4070.

[25] D.H. Thomas, P. Looney, R. Steel, N. Pelekasis, W.N. McDicken, T. Anderson, V. Sboros, Acoustic detection of microbubble resonance, Appl. Phys. Lett. 94 (2009) 243902.

[26] A.A. Doinikov, P.A. Dayton, Maxwell rheological model for lipid-shelled ultrasound microbubble contrast agent, J. Acoust. Soc. Am. 121 (2007) 33313340 .

[27] A. Prosperetti, A. Lezzi, Bubble dynamics in a compressible liquid. Part 1. Firstorder theory, J. Fluid Mech. 168 (1986) 457-478.

[28] C. Truesdell, W. Noll, The non-linear field theories of mechanics, in: S. Flügge (Ed.), Encyclopedia of Physics, Springer-Verlag, Berlin, 1965.

[29] S. Hilgenfeltdt, D. Lohse, M. Zomack, Response of bubbles to diagnostic ultrasound: a unifying theoretical approach, Eur. Phys. J. B 4 (1998) 247

[30] L. Hoff, Acoustic Characterization of Contrast Agents for Medical Ultrasound Imaging, Kluver, Dordrecht, 2001.

[31] S.M. van der Meer, B. Dollet, D.E. Goertz, N. de Jong, M. Versluis, D. Lohse, Surface modes of ultrasound contrast agent microbubbles, IEEE Ultrason. Symp. (2006) 112-115.

[32] B. Dollet, S.M. van der Meer, V. Garbin, N. de Jong, D. Lohse, M. Versluis, Nonspherical oscillations of ultrasound contrast agent microbubbles, Ultrasound Med. Biol. 34 (2008) 1465-1473. 
[33] K. Tsiglifis, N.A. Pelekasis, Parametric stability and dynamic buckling of an encapsulated microbubble subject to acoustic disturbances, Phys. Fluids 23 (2011) 012102

[34] S. Grossmann, S. Hilgenfeldt, D. Lohse, M. Zomack, Sound radiation of a $3 \mathrm{MHz}$ driven gas bubble, J. Acoust. Soc. Am. 102 (1997) 1223

[35] O.S. Mukdadi, Hyong-Bum Kim, J. Hertzberg, R. Shandas, Numerical modeling of microbubble backscatter to optimize ultrasound particle image velocimetry imaging: initial studies, Ultrasonics 42 (2004) 1111-1121.

[36] M. Kaya, S. Feingold, K. Hettiarachchi, A.P. Lee, P.A. Dayton, Acoustic responses of monodisperse lipid encapsulated microbubble contrast agents produced by flow focusing, Bubble Sci. Eng. Technol. 2 (2) (2010) 33-40.
[37] A. Bouakaz, S. Frigstad, F.J. Ten Cate, N. de Jong, Improved contrast to tissue ratio at higher harmonics, Ultrasonics 40 (2002) 575-578.

[38] K. Cheung, O. Couture, P.D. Bevan, E. Cherin, R. Willians, P.B. Burns, F.S. Foster, In vitrocharacterization of the subharmonic ultrasound signal from Definity microbubbles at high frequencies, Phys. Med. Biol. 53 (2008) 1-15.

[39] J. Sijl, B. Dollet, M. Overvelde, V. Garbin, T. Rozendal, N. de Jong, D. Lohse, M. Versluis, Subharmonic behavior of phospholipid-coated ultrasound contrast agent microbubbles, J. Acoust. Soc. Am. 128 (2010) 3239-3252.

[40] A.A. Doinikov, A. Bouakaz, Review of shell models for contrast agent microbubbles, IEEE Trans. Ultrason. Ferroelect. Freq. Control 58 (5) (2011) 981-993. 